\title{
Modeling of fluid resonance in-between two floating structures in close proximity*
}

\author{
Chao-bang YAO, Wen-cai DONG \\ (Department of Naval Architecture Engineering, Naval University of Engineering, Wuhan 430033, China) \\ E-mail: hgycb2004111@163.com; haigongdwc@163.com
}

Received Jan. 20, 2015; Revision accepted Aug. 22, 2015; Crosschecked Nov. 27, 2015

\begin{abstract}
In this study, we conducted numerical simulations of fluid resonance in-between two floating structures based on potential theory assessing the effect of fluid viscosity by including the artificial damping force. The numerical results of two adjacent Barges systems and Barge \& Wigley systems were compared with experimental data of those of the viscous fluid model based on Reynolds average Navier-Stokes equations (RANSE). It can be observed that the conventional potential flow model (without artificial damping force) significantly over-estimated the wave height and forces around the resonant frequencies. Results of the present method with an appropriate damping coefficient supported the available data, confirming the importance of the viscous damping effect on strong hydrodynamic interaction between the floating structures. Furthermore, influences of lateral clearances, wave heading angles, and ships' motions on the wave surface elevations were analyzed. Validation and application of methods to estimate the fluid resonant frequencies and modes were also conducted. Generally speaking, Molin's simplified theory can give an accurate estimation of resonant frequencies and serve as a practical tool to analyze the fluid resonant phenomena of gaps in-between a two Barge system and Wigley \& Barge system in close proximity.
\end{abstract}

Key words: Fluid resonance, Water wave, Boundary element method, Artificial damping forces, Sloshing mode doi: $10.1631 /$ jzus.A1500017

Document code: A

CLC number: U661.32

\section{Introduction}

Hydrodynamic interaction effect becomes important for multiple floating structures arranged side by side with small separation, which are often encountered in marine and offshore practices, such as the very large floating structure (VLFS) involving many separated modules and floating production storage and floating facility (FPSO), and a large vessel berthing in front of wharf. The actual geometry is such that 3D effects can be important, and nearresonant standing waves can also exist along the length of the gap.

\footnotetext{
* Project supported by the National Natural Science Foundation of China (No. 50879090), the Key Research Program of Hydrodynamics of China (No. 9140A14030712JB11044), and the Innovation Research Foundation for PhD Candidates of Naval University of Engineering (No. HGBSJJ2013007), China

(D) ORCID: Chao-bang YAO, http://orcid.org/0000-0001-9092-7659

(c) Zhejiang University and Springer-Verlag Berlin Heidelberg 2015
}

This interesting phenomenon has, understandably, attracted great attention. The first numerical investigation of the 3D problem was probably that of Newman and Sclavounos (1988), and more recently the interactions between multiple closely spaced bodies have been further studied by other researchers (Kashiwagi et al., 2005; Kashiwagi, 2007; Koo and Kim, 2005; Pauw et al., 2007; Lewandowski, 2008; Sun et al., 2008; 2010; Molin et al., 2009; Chen, 2011). They have all shown that, when the multiple floating structures with narrow gaps are subjected to water waves, fluid resonance may take place. This can lead to the vertical oscillations of the mean surface having amplitudes substantially larger than the incident wave in narrow gaps and the increase of wave forces around the resonant frequencies. Thus, practical interest is confined not only to the forces and motions of the closely spaced vessels but also to the free surface elevations. 
A number of publications have been recently released concerning the resonant motion of the wave field in the confided zone between two floating bodies. Unlike the resonant response of body's motion associated with the balance of inertia and stiffness loads, this resonant kinematics of fluid is annulled or amplified by complex scattering between bodies (Chen, 2011). Within the framework of the classical linear potential theory (for example aspects based on the boundary integral method, 3D model in frequency and time domain), there is no limit in terms of predicting wave elevations at the free surface. This flow model tends to overestimate the wave amplitudes in narrow gaps because of the physical energy resulting from fluid viscosity, vortex shedding, and turbulence which cannot be taken into account in the context of potential flow theory. This unrealistic fluid motion magnifies the wave loads on the bodies. To contain the wave motion within a realistic level, Buchner et al. (2001) developed a method consisting of placing a rigid lid on the gap in between the two bodies. The unrealistic wave kinematics is thus suppressed. In fact, no wavy elevation is possible under this rigid lid and noticeable perturbation around the ends of the lid can be observed due to the diffraction effect. To make wavy motion possible on the lid, Newman (2003) rendered the lid flexible using a set of basic functions of Chebychev polynomials. The deformation of the flexible mat (equal to the free surface elevation) is then reduced by introducing a damping coefficient. Chen (2011) proposed the introduction of a linear dissipation term in the free surface equation, which was referred as fairly perfect fluid potential theory. It has been shown that the technique of incorporating artificial resistance into the conventional potential flow model may work well in limiting the amplitude of water oscillations in narrow gaps. In addition, the vortex method employed to overcome the difficulties produced by a conventional potential model gives reasonable results, but it has only been used in 2D case.

The resonant frequency is another key concern. It has been noted by other researchers that this problem is closely related to resonant phenomenon of the moonpool, which was comprehensively investigated by Molin (2001) and Molin et al. (2002). Other works which pertain to this problem are those of Miao et al. (2000), Zhu et al. (2005; 2008), Saitoh et al. (2006), Iwata et al. (2007), Wang and Wu (2008), and Lu et al. (2011a; 2011b).
The present study focuses on the fluid resonances between two floating structures based on fairly perfect fluid potential theory (taking the effect of fluid viscosity into consideration). Additionally this paper also analyzes methods used to estimate the fluid resonant frequencies. Furthermore, the validation and practical application of these methods for ship hulls are investigated.

\section{Numerical model}

\subsection{Potential flow considering the effect of fluid viscosity}

In this section we study floating bodies with or without forward speed on a free surface and in the presence of incident propagative waves. The reference system of Cartesian coordinates is defined by letting $(x, y)$ plane coincide with the mean free surface and a positive and upwards $z$-axis. The fluid is assumed to be incompressible and inviscid while the fluid motion is irrotational. Under the assumptions of a perfect fluid, the fluid velocity $\boldsymbol{V}=\left(V_{1}, V_{2}, V_{3}\right)$ can be expressed as $\boldsymbol{V}=\nabla \Phi$, the gradient of a scalar potential $\Phi$, which satisfies the Laplace equation according to mass conservation. The fluid is under the action of gravity. This gravitational field, an internal force,

$$
f=-\mu V
$$

is assumed to apply to the fluid particle as well. The parameter $\mu$ is assumed to be positive and small, and this force is proportional to the magnitude of fluid velocity but moving in the opposite direction. Although it plays the same role of dampening fluid motion and dissipating energy as that of fluid viscosity, it does not introduce any vortices so the existence of velocity potential is safeguarded. The inviscid and irrotational fluid with the artificial damping force is called by Guevel (1982) the fairly perfect fluid. The momentum equation for the fairly perfect fluid is slightly modified and the associated Bernoulli equation is expressed as (Chen, 2011)

$$
P / \rho+g z+\Phi_{t}+\nabla \Phi \cdot \nabla \Phi / 2+\mu \Phi=C(t),
$$

where $\rho$ is the water density, $g$ is the gravitational 
acceleration, $P$ is the dynamic pressure of ship hull, and $C(t)$ is a constant number.

At the first-order problem, combining both the dynamic and kinematic free surface conditions yields a compound form:

$$
g \Phi_{z}+\Phi_{t t}+\mu \Phi=0
$$

Furthermore, the free surface elevation is given by

$$
\eta=-\left(\Phi_{t}+\mu \Phi\right) / g
$$

\subsection{Velocity potential}

The regular wave is coming from a direction with an angle $\beta$, which is the angle between the positive $x$-axis and the incident wave direction. Thus, $180^{\circ}$ means it is facing head-on towards the sea. The incident potential $\Phi_{0}$ is given as

$$
\begin{aligned}
& \Phi_{0}(x, y, z, t)=\phi_{0} \mathrm{e}^{-\mathrm{i} \omega_{\mathrm{e}} t}, \\
& \phi_{0}=-\frac{\mathrm{i} g \varsigma}{\omega_{0}} \mathrm{e}^{k_{0} z} \mathrm{e}^{k_{0}(x \cos \beta+y \sin \beta)},
\end{aligned}
$$

where $k_{0}=\omega_{0}^{2} / g$ is the wave number of the incident wave, $\omega_{0}$ and $\varsigma$ are the frequency and amplitude, respectively, and $\omega_{\mathrm{e}}$ is the encounter frequency.

The velocity potential $\Phi_{T}(x, y, z, t)$ can be expressed as

$$
\Phi_{T}(x, y, z, t)=\phi_{\mathrm{s}}(x, y, z)+\Phi(x, y, z, t),
$$

where $\phi_{\mathrm{s}}$ and $\Phi$ are the steady disturbance potential and unsteady potential, respectively. In the firstorder problem, all unsteady motions are assumed to be sinusoidal in time with the encounter frequency $\omega_{\mathrm{e}}$, and by linear decomposition the unsteady potential $\Phi$ can be expressed as

$$
\Phi(x, y, z, t)=\Phi_{R}+\Phi_{7}+\Phi_{0}=\left(\phi_{R}+\phi_{7}+\phi_{0}\right) \mathrm{e}^{-\mathrm{i} \omega_{\mathrm{e}} t}
$$

where $\Phi_{R}$ and $\Phi_{7}$ are the coupled radiation and diffraction potentials in the field, and $\phi_{R}$ and $\phi_{7}$ are the time-independent aspects.

\section{Numerical methods}

\subsection{Integral equations}

Presenting potential flow theory directly uses authentic equations, and involves the energy dissipating via the introduction of the damping force as illustrated by Eq. (1). In most cases, the damping coefficient $\mu$ is set to zero without affecting the results. However, this damping term must play an important role in predicting the wave field within the confined zone, i.e., wave elevation of the gap in-between two floating structures. In these cases, the radiation and diffraction potential $\phi_{j}$ can be expressed as (Chen, 2011)

$$
\phi_{j}(p)=\iint_{S} \sigma G(p, q) \mathrm{d} S, \text { with } S=S_{a} \cup S_{b} \cup S_{F^{\prime}} \cup S_{F_{m}},
$$

and the integral equations to determine the source distribution $\sigma$ are

$$
\begin{aligned}
& 2 \pi \sigma(p)+\iint_{S} \mathrm{~d} S \sigma(q) G_{n}(p, q)=v_{n}, p \subset S_{a} \cup S_{b}, \\
& 4 \pi \sigma(p)-\iint_{S} \mathrm{~d} S \sigma(q) G_{n}(p, q)=0, p \subset S_{F^{\prime}} \\
& 4 \pi \sigma(p)+\mathrm{i} \varepsilon k_{0} \iint_{S} \mathrm{~d} S \sigma(q) G(p, q)=0, p \subset S_{F_{m}}
\end{aligned}
$$

where $\varepsilon=\mu / \omega_{0}, S_{a}$ and $S_{b}$ are the mean wetted surface of ship- $a$ and ship- $b$, respectively, $S_{F^{\prime}}$ is the interior free surface of ship hull (also named as lid) and $S_{F_{m}}$ is the entire free surface; $v_{n}=n_{a j}, n_{b j}(j=1,2, \ldots, 6)$ represent radiation problems, and $v_{n}=-\partial \phi_{0} / \partial n$ signify diffraction problem, with $n_{a j}, n_{b j}$ as the normal vectors of ship- $a$ and ship- $b$, which are oriented positively towards the fluid.

Thus, it can be known that $\sigma=0$ if $\varepsilon=0$. A nonzero value of $\varepsilon$ was applied only in the zone where the fluid kinematics is susceptible to behaving in a volatile manner, so that the discretization of free surface is confined to the gap in-between the two ships.

Here, the pulsating source Green's function is employed in Eqs. (9)-(12), which can be expressed as

$$
G(p, q)=-\left(1 / r+1 / r_{1}\right)-2 f\left[R_{0}(h, v)+\mathrm{i} \pi J_{0}(h) \cdot \mathrm{e}^{v}\right],
$$




$$
\begin{aligned}
\frac{\partial G(p, q)}{\partial x}= & {\left[\left(\frac{R}{r^{3}}\right)+\left(\frac{R}{r_{1}^{3}}\right)+2 f^{2}\left(R_{1}(h, v)\right.\right.} \\
& \left.\left.+\mathrm{i} \pi J_{1}(h) \cdot \mathrm{e}^{v}\right)\right]\left(\frac{x-\xi}{R}\right), \\
\frac{\partial G(p, q)}{\partial y}= & {\left[\left(\frac{R}{r^{3}}\right)+\left(\frac{R}{r_{1}^{3}}\right)+2 f^{2}\left(R_{1}(h, v)\right.\right.} \\
& \left.\left.+\mathrm{i} \pi J_{1}(h) \cdot \mathrm{e}^{v}\right)\right]\left(\frac{y-\eta^{\prime}}{R}\right), \\
\frac{\partial G(p, q)}{\partial z}= & \frac{z-\zeta}{r^{3}}+\frac{z+\zeta}{r_{1}^{3}} \\
& -2 f^{2}\left(1 / d+R_{0}(h, v)+\mathrm{i} \pi J_{0}(h) \cdot \mathrm{e}^{v}\right),
\end{aligned}
$$

where

$$
\begin{aligned}
& r=\left[(x-\xi)^{2}+\left(y-\eta^{\prime}\right)^{2}+(z-\zeta)^{2}\right]^{1 / 2} / L, \\
& r_{1}=\left[(x-\xi)^{2}+\left(y-\eta^{\prime}\right)^{2}+(z+\zeta)^{2}\right]^{1 / 2} / L, \\
& R=\left[(x-\xi)^{2}+\left(y-\eta^{\prime}\right)^{2}\right]^{1 / 2} / L, \\
& f=\frac{\omega^{2} L}{g}, \\
& h=f \cdot R, \\
& v=f(z+\zeta) / L, \\
& d=\left(h^{2}+v^{2}\right)^{1 / 2} .
\end{aligned}
$$

$p(x, y, z)$ and $q\left(\xi, \eta^{\prime}, \zeta\right)$ are the coordinates for field point and source point, respectively. The variables $R, r, r_{1}$ and $h, v, d$ are non-dimensional in terms of the reference lengths $L$ and $g / \omega^{2}$, respectively. $J_{0}(h)$ and $J_{1}(h)$ are the usual Bessel functions of the first kind. $R_{0}(h, v)$ and $R_{1}(h, v)$ are real functions to be defined by four different series expansions and one integral representation submitted by Telste and Noblesse (1986). As for ship with forward speed, the translating-pulsating source Green function can be used (Yao and Dong, 2014).

\subsection{Wave exciting forces and hydrodynamic coefficients}

Considering the unsteady state of incident waves, omitting the high order terms and static water pressure, the linear hydrodynamic pressure equation becomes

$$
P=\rho \mathrm{i} \omega_{\mathrm{e}}\left(1-\frac{\mu}{\mathrm{i} \omega_{\mathrm{e}}}\right) \phi \mathrm{e}^{-\mathrm{i} \omega_{\mathrm{e}} t} .
$$

By replacing the potential $\phi$ by radiation potentials, the hydrodynamic pressure induced by oscillation motion can be obtained. Then the related hydrodynamic coefficients for added mass and damping can be obtained as

$$
\left\{\begin{array}{l}
-A_{a a i j} \omega_{\mathrm{e}}^{2}-B_{a a i j} \omega_{\mathrm{e}}=-\rho \mathrm{i} \omega_{\mathrm{e}} \iint_{S_{a}}\left(1-\frac{\mu}{\mathrm{i} \omega_{\mathrm{e}}}\right) \phi_{a a j} n_{a i} \mathrm{~d} s, \\
-A_{a b i j} \omega_{\mathrm{e}}^{2}-B_{a b i j} \omega_{\mathrm{e}}=-\rho \mathrm{i} \omega_{\mathrm{e}} \iint_{S_{b}}\left(1-\frac{\mu}{\mathrm{i} \omega_{\mathrm{e}}}\right) \phi_{a b j} n_{b i} \mathrm{~d} s, \\
-A_{b a i j} \omega_{\mathrm{e}}^{2}-B_{b a i j} \omega_{\mathrm{e}}=-\rho \mathrm{i} \omega_{\mathrm{e}} \iint_{S_{a}}\left(1-\frac{\mu}{\mathrm{i} \omega_{\mathrm{e}}}\right) \phi_{b a j} n_{a \mathrm{i}} \mathrm{d} s, \\
-A_{b b i j} \omega_{\mathrm{e}}^{2}-B_{b b i j} \omega_{\mathrm{e}}=-\rho \mathrm{i} \omega_{\mathrm{e}} \iint_{S_{b}}\left(1-\frac{\mu}{\mathrm{i} \omega_{\mathrm{e}}}\right) \phi_{b b j} n_{b i} \mathrm{~d} s,
\end{array}\right.
$$

where $A_{a a i j}, B_{a a i j}$, and $A_{a b i j}, B_{a b i j}$ are the added mass and damping coefficients on ship- $a$ which result from the motion of ship- $a$ and ship- $b$, respectively; $A_{b a i j}, B_{b a i j}$, and $A_{b b i j}, B_{b b i j}$ are the added mass and damping coefficients on ship- $b$ caused by the motion of ship- $a$ and ship- $b$, respectively.

Correspondingly, the incident wave forces and coupled diffraction forces of each ship can be defined as

$$
\left\{\begin{array}{l}
F_{\mathrm{w} a i}(t)=f_{\mathrm{w} a i} \mathrm{e}^{-\mathrm{i} \omega_{\mathrm{e}} t} \\
=-\rho \mathrm{i} \omega_{\mathrm{e}} \mathrm{e}^{-\mathrm{i} \omega_{\mathrm{e}} t} \iint_{S_{a}}\left(1-\frac{\mu}{\mathrm{i} \omega_{\mathrm{e}}}\right)\left(\phi_{7}+\phi_{0}\right) n_{a i} \mathrm{~d} s, \\
F_{\mathrm{w} b i}(t)=f_{\mathrm{w} b i} \mathrm{e}^{-\mathrm{i} \omega_{\mathrm{e}} t} \\
=-\rho \mathrm{i} \omega_{\mathrm{e}} \mathrm{e}^{-\mathrm{i} \omega_{\mathrm{e}} t} \iint_{S_{b}}\left(1-\frac{\mu}{\mathrm{i} \omega_{\mathrm{e}}}\right)\left(\phi_{7}+\phi_{0}\right) n_{b i} \mathrm{~d} s,
\end{array}\right.
$$

where $f_{\mathrm{w} a i}$ and $f_{\mathrm{w} b i}$ are the waves exciting the force of ship- $a$ and ship- $b$, respectively.

\section{Numerical results of twin barge ships}

\subsection{Configuration and properties}

The configuration consists of two side-by-side rectangular boxes of the same dimension in meters $(L \times B \times T=2.47 \times 0.6 \times 0.18)$ with mechanical properties $\left(z_{G}=0.02 \mathrm{~m}\right.$ and gyration radii $=0.187 / 0.527 /$ 
0.527). The mesh of barges and the whole gap meshed as the damping zone are presented in Fig. 1.

Cases of different lateral clearance, the boxes fully fixed or freely floating, and the different wave heading angles are considered in order to assess the influence of those parameters on the hydrodynamics of barges.

\subsection{Methods to determine dissipate parameter $\varepsilon$}

The free floating model tests were realized in Oceanide and reported by Rippol (2004). The free surface wave elevation was measured at the center of the gap (marked as $P$ in Fig. 1).

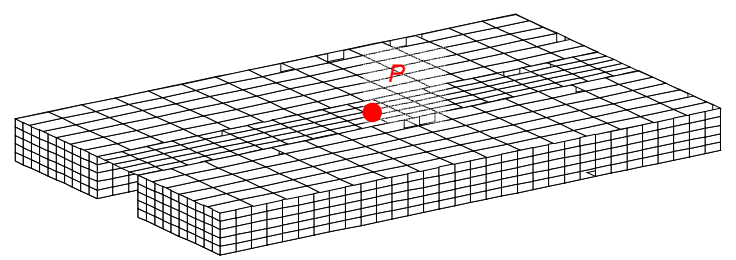

Fig. 1 Mesh of two barges and damping zone

The dissipate parameter $\varepsilon$ can be determined by comparing numerical results of potential theory with internal damping force to those of the experiments. For the two barges introduced above, the wave elevation results (i.e., per $1 \mathrm{~m}$ incident wave amplitude) of numerical computation with values of parameter $\varepsilon=0$ and 0.016 are drawn in Fig. 2 together with those of measurements and numerical calculations by Chen (2011). As is evident, the results of conventional potential method (without lid or with lid, $\varepsilon=0$ ) are much larger than those measured, while the results with $\varepsilon=0.016$ support the measurements of around the resonant frequencies, confirming the importance of viscous damping effect on the strong interaction between two adjacent floating structures.

Another convenient way to determine the dissipate parameter is by comparing the numerical results of potential and viscous fluid theory. Here, viscous results (referred to as Reynolds average NavierStokes equations (RANSE)) are obtained by using the numerical wave tank which has been developed and based on the commercial software Fluent, at the core of which are the Navier-Stokes equations and turbulent models. Additionally, the volume of fluid method is used to capture the free surface.

The results of numerical computation with val- ues of parameter $\varepsilon=0,0.008,0.016$, and 0.032 and viscous model are presented in Fig. 3 for $D_{y}=0.8 \mathrm{~m}$, $0.9 \mathrm{~m}$ at a heading sea, and $D_{y}=0.716 \mathrm{~m}$ at $\beta=150^{\circ}$. As shown in Fig. 3, the conventional potential flow model always significantly over-predicts the wave height in narrow gap around the resonant frequency. However, the results of present potential theory with $\varepsilon=0.016$ show good correlation with those of RANSE.

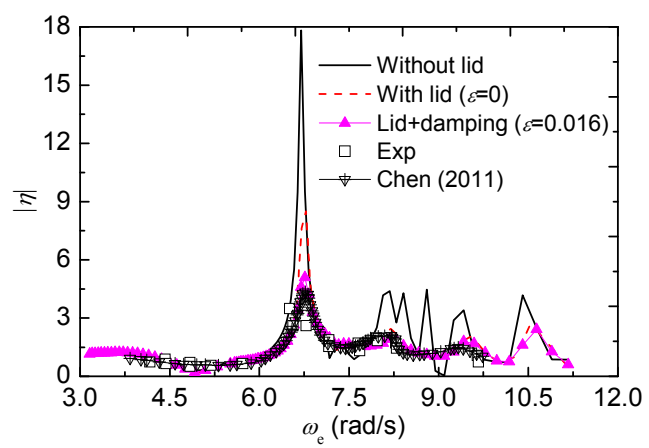

Fig. 2 Wave elevations in the gap center of two barges: total wave elevation for free floating bodies for $D_{y}=0.716 \mathrm{~m}$

\subsection{First-order wave elevation in the gap}

The motion of the free surface in the gap between two side-by-side barges is now explored using the code which has been developed using the theory introduced above. Results are presented as response amplitude operators (RAOs) of the free-surface amplitude (i.e., per $1 \mathrm{~m}$ incident wave amplitude) at the center of gap. According to the above analysis, the damping coefficient is set to a constant value of 0.016 .

4.3.1 Influence of lateral clearance on wave elevation

Firstly, the conditions of the twin barges arranged side-by-side with different spacings are considered. Here, three typical centerline distances of $0.716 \mathrm{~m}, 0.8 \mathrm{~m}$, and $0.9 \mathrm{~m}$ are utilized.

RAOs of wave elevation in the gap are shown in Fig. 4 under beam seas. It can be observed that there are many resonant peaks. The frequencies of peaks depend on the spacing, and they shift to higher frequencies with the decrease of spacing, i.e., the first peak frequency is $4.94 \mathrm{rad} / \mathrm{s}, 5.34 \mathrm{rad} / \mathrm{s}$, and $5.82 \mathrm{rad} / \mathrm{s}$ for $D_{y}=0.9 \mathrm{~m}, 0.8 \mathrm{~m}$, and $0.716 \mathrm{~m}$, respectively. In addition, the magnitudes of wave elevation decrease by up to $23.4 \%$ with the increase 
of investigated spacings and the maximum peak value is about 4-5 times the amplitude of the incident wave. The resonant frequencies and their relationship to the sloshing and piston mode will be discussed in details in section 4.4.

4.3.2 Influence of wave heading angle on wave elevation

The influence of wave heading angle on the fluid resonance is determined by the fixed lateral distance of $D_{y}=0.716 \mathrm{~m}$ and four wave heading angles, namely, $\beta=90^{\circ}, 120^{\circ}, 150^{\circ}$, and $180^{\circ}$. Fig. 5 shows the variation of wave elevations with incident wave frequency $\omega_{\mathrm{e}}$.
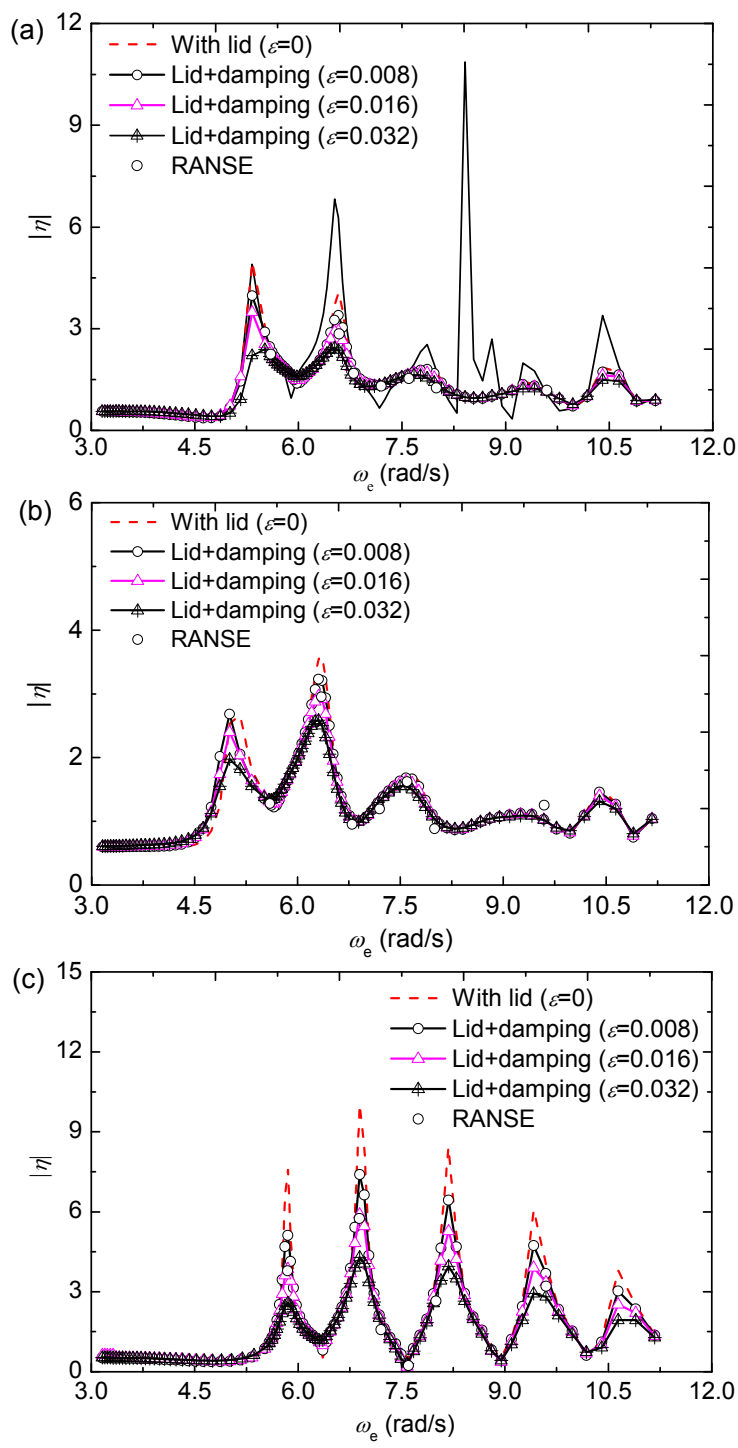

Fig. 3 Wave elevation in the center gap of two barges Diffraction wave elevation for $D_{y}=0.8 \mathrm{~m}$ (a), $D_{y}=0.9 \mathrm{~m}$ (b), and $D_{y}=0.716 \mathrm{~m}, \beta=150^{\circ}$ (c)

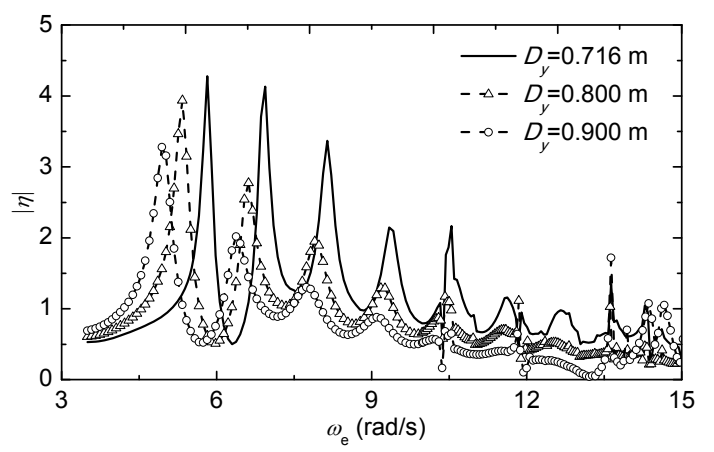

Fig. 4 Effect of spacing on diffraction wave elevation in the gap in beam seas

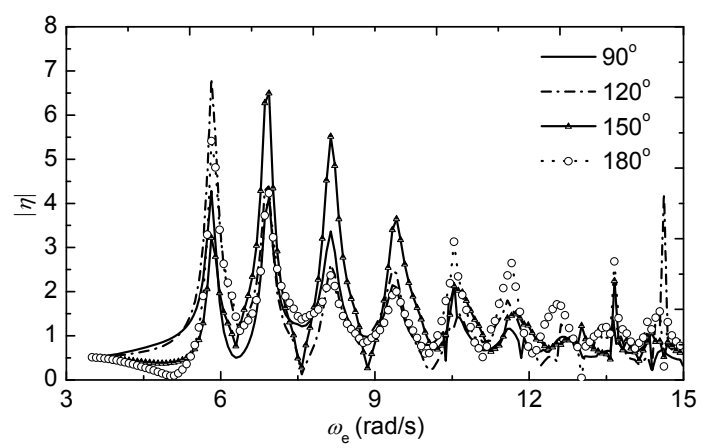

Fig. 5 Effect of wave direction on diffraction wave elevation in the gap with $D_{y}=0.716 \mathrm{~m}$

Numerical results shown in Fig. 5 confirm that many resonant peaks still exist and the locations of peaks in different wave directions are similar, but not identical. As established by Sun et al. (2010), the difference is due to the fact that in beam seas the responses are essentially standing waves, while in head seas these are superposed on a progressive wave, including the effects of incident and diffraction waves along the gap.

It also can be seen that the first peak wave elevation at $\beta=120^{\circ}$ is much greater than that of other heading angles; meanwhile, the second peak wave elevation at $\beta=150^{\circ}$ is higher than that of others. This fact indicates that the wave elevation of the gap inbetween two barges considered here is more significant under oblique seas, and that these situations should be avoided for practical operations in seaways.

\subsubsection{Influence of barge motion on wave elevation}

Fig. 6 shows the free surface amplitude RAO for cases with both barges fixed and both freely floating. The spacing is $0.716 \mathrm{~m}$ and the barge ships are in beam seas. 
It can be observed that the locations of the peaks shift depending on whether the barge is fixed or freely floating. The frequency corresponding to the first resonant peak of cases where both barges are fixed is lower, i.e., $4.94 \mathrm{rad} / \mathrm{s}$ for both barges fixed whilst it is $6.06 \mathrm{rad} / \mathrm{s}$ when both barges are floating freely; meanwhile, the peak magnitudes of the wave elevation are substantially smaller. The reason is that the components of total wave elevation of these two cases are different. As for the fixed situation, only incident and diffraction waves are involved, whilst with the incident wave, radiation waves due to oscillation of both barges and diffraction wave are included for free floating cases.

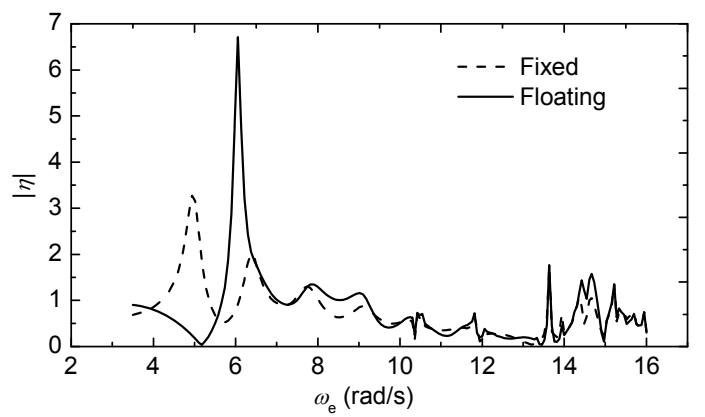

Fig. 6 Effect of box motions on the total wave elevation in the gap in beam seas with $D_{y}=0.716 \mathrm{~m}$

However, with the increase of frequency of incident waves, discrepancies between two cases become small and almost the same when $\omega_{\mathrm{e}}>10 \mathrm{rad} / \mathrm{s}$.

The sway and heave motions of the barges are presented in Fig. 7. Ship- $a$ and ship- $b$ are at the shadow and weather side, respectively. An evident sheltering effect by the weather side ship on the other ship can be observed.

\subsection{Analysis of resonant frequencies and associ- ated Eigen modes}

As discussed by Newman and Sclavouns (1988) and Molin et al. (2002), the problem of fluid resonance in-between two barges arranged side-by-side with a small gap can be treated as an open-ended moonpool. However, the proper boundary condition (the no-flow Neumann condition or the zero pressure Dirichlet condition) for the ended boundary still requires further discussion.

Based on the assumption of potential flow theory, the flow within the moonpool is expressed in terms of Eigen function expansions, and matched to the flow below the plate by means of an integral equation. As for the flow in the gap between the two parallel barges, the problem can be treated in a similar fashion. Here, Neumann or Dirichlet boundary conditions are taken into consideration.
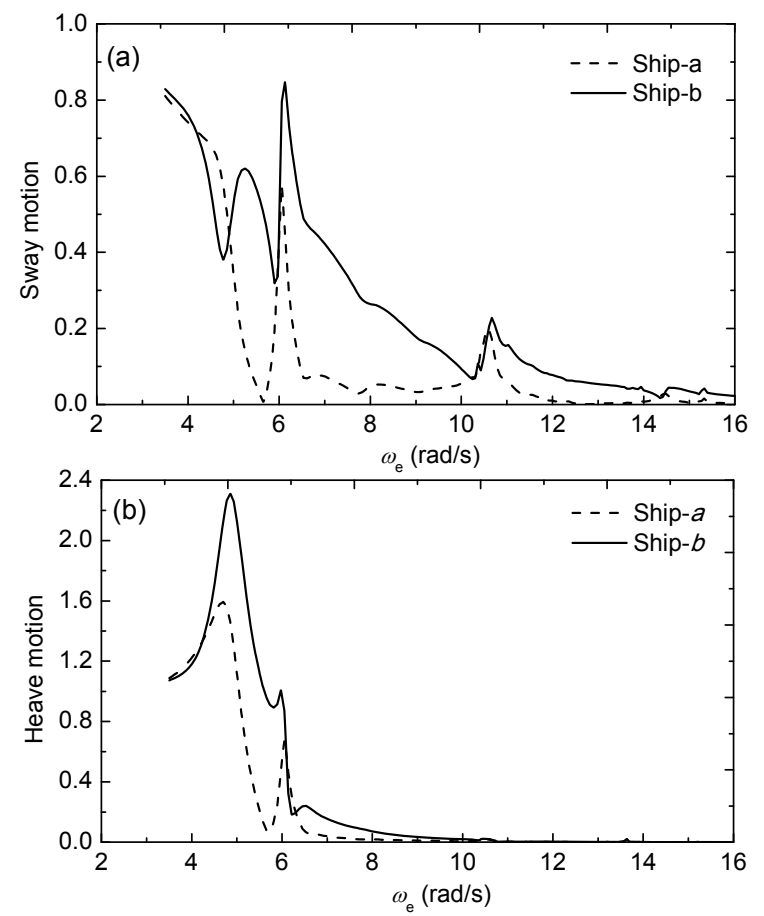

Fig. 7 Sway motion (a) and heave motion (b) in beam seas with $D_{y}=0.716 \mathrm{~m}$

As pointed out by Molin (2001), it is easy to see that the velocity potential $\Phi$ in the fluid field satisfies the Laplace equation and linear free surface conditions. If it is assumed to be periodic at frequency $\omega$, then it can be written in the form:

$\Phi=$

$\sum_{m=0}^{\infty} \sum_{n=0}^{\infty} f_{m}(x) g_{n}(y)\left[C_{m n} \cosh \left(v_{m n} z\right)+D_{m n} \sinh \left(v_{m n} z\right)\right] \cos (\omega t)$.

The functions $f_{m}$ and $g_{n}$ are determined by the no-flow boundary conditions on the walls and openend boundary conditions, which yield $f_{m}(x)=\sin \left(\lambda_{m} x\right)$, $g_{n}(y)=\cos \left(\mu_{n} y\right)$ for the Dirichlet condition and $f_{m}(x)=\cos \left(\lambda_{m} x\right), g_{n}(y)=\cos \left(\mu_{n} y\right)$ for the Neumann condition, with $\lambda_{m}=m \pi / L_{\mathrm{g}}, \mu_{n}=n \pi / B_{\mathrm{g}}$, and $\lambda_{m}^{2}+\mu_{n}^{2}=v_{m n}^{2}$, $L_{\mathrm{g}}$ and $B_{\mathrm{g}}$ as the length and width of the gap, respectively. The coefficients $C_{m n}$ and $D_{m n}$ are obtained by imposing the free-surface boundary condition and 
the coupling condition. The coupling condition is satisfied by using the Galerkin procedure: it is multiplied by $f_{m^{\prime}}(x) g_{n^{\prime}}(y)$ and integrated over $\left(0<x<L_{\mathrm{g}}\right.$, $0<y<B_{\mathrm{g}}$ ) (details can be seen in (Molin, 2001)). Consequently, an eigenvalue problem for the resonant frequencies $\omega_{m n}$ can be solved. As pointed out by Molin (2001), when using single mode approximations, the general form of $\omega_{m n}$ is (with $H$ the draught of ship hull):

$$
\omega_{m n}^{2}=g v_{m n} \frac{1+J_{m n} \tanh \left(v_{m n} H\right)}{J_{m n}+\tanh \left(v_{m n} H\right)}
$$

where

$$
\begin{aligned}
& J_{m n}=\frac{v_{m n}}{2 \pi} \\
& \frac{\int_{0}^{L_{\mathrm{g}}} \int_{0}^{L_{\mathrm{g}}} \int_{0}^{B_{\mathrm{g}}} \int_{0}^{B_{\mathrm{g}}}(1 / R) f_{m}(x) f_{m}\left(x^{\prime}\right) g_{n}(y) g_{n}\left(y^{\prime}\right) \mathrm{d} x \mathrm{~d} x^{\prime} \mathrm{d} y \mathrm{~d} y^{\prime}}{\int_{0}^{L_{\mathrm{g}}} \int_{0}^{B_{\mathrm{g}}}\left(f_{m}(x)\right)^{2}\left(g_{n}(y)\right)^{2} \mathrm{~d} x \mathrm{~d} y} .
\end{aligned}
$$

In the general case of arbitrary $m$ and $n$, Molin (2001) showed that the quadruple integral may be reduced to a double integral. In the special case of $n=0$ (no variation of wave elevation across the gap), a simpler single integral can be derived based on the residue theorem. Thus, $J_{m 0}$ for Neumann conditions can be written as (Molin, 2001)

$$
\begin{aligned}
J_{m 0}^{\mathrm{N}} & =\frac{2}{n \pi^{2} r}\left\{\int_{0}^{1} \frac{r^{2}}{u^{2} \sqrt{u^{2}+r^{2}}}[1+(u-1) \cos (n \pi u)\right. \\
& \left.\left.-\frac{\sin (n \pi u)}{n \pi}\right] \mathrm{d} u+\frac{1}{\sin \theta_{0}}-1\right\} .
\end{aligned}
$$

$J_{m 0}$ for the Dirichlet condition can be expressed as (Molin et al., 2002)

$$
\begin{aligned}
J_{m 0}^{\mathrm{D}} & =\frac{2}{n \pi^{2} r}\left\{\int_{0}^{1} \frac{r^{2}}{u^{2} \sqrt{u^{2}+r^{2}}}[1+2 u+(u-1) \cos (n \pi u)\right. \\
& \left.\left.-\frac{3}{n \pi} \sin (n \pi u)\right] \mathrm{d} u-\frac{1}{\sin \theta_{0}}+1+2 r \ln \frac{1+\cos \theta_{0}}{1-\cos \theta_{0}}\right\},
\end{aligned}
$$

where $r=B_{\mathrm{g}} / L_{\mathrm{g}}$ and $\tan \theta_{0}=1 / r$. For the general case $n>0$, a numerical approach proposed by Sun et al. (2010) is adopted, for which the numerator in Eq. (22) should be written as

$$
\begin{aligned}
I & =\int_{0}^{L_{\mathrm{g}}} \int_{0}^{B_{\mathrm{g}}}\left\{f_{m}(x) g_{n}(y) \int_{0}^{L_{\mathrm{g}}} \int_{0}^{B_{\mathrm{g}}} \frac{\mathrm{d} x^{\prime} \mathrm{d} y^{\prime}}{R}\right. \\
& \left.+\int_{0}^{L_{\mathrm{g}}} \int_{0}^{B_{g}}\left[f_{m}\left(x^{\prime}\right) g_{n}\left(y^{\prime}\right)-f_{m}(x) g_{n}(y)\right] \frac{\mathrm{d} x^{\prime} \mathrm{d} y^{\prime}}{R}\right\} f_{m}(x) g_{n}(y) \mathrm{d} x \mathrm{~d} y .
\end{aligned}
$$

The inner double integral of $1 / R$ can be evaluated analytically. The second inner double integral may be computed numerically. In a comparable fashion to the numerical method of Sun et al. (2010), the high order Gaussian quadrature is used, for which the integration points and weights are calculated by the algorithm proposed by Recktenwald (2000).

The convergence of this numerical approach is corroborated by Sun et al. (2008). In order to check the validation of the present numerical integration algorithm code, $J_{m 0}^{\mathrm{N}}$ and $J_{m 0}^{\mathrm{D}}$ are calculated with $L_{\mathrm{g}}=2.87 \mathrm{~m}, B_{\mathrm{g}}=0.116 \mathrm{~m}$. Tables 1 and 2 show the results for the symmetric modes in the cases. It can be seen from the two tables that $24 \times 24$ quadrature (indicated as Gauss 24 in the table) is accurate to within about 5D-6D.

Table 1 Convergence of $J_{m 0}^{\mathrm{N}}$ factors with Gauss integration order

\begin{tabular}{lcccc}
\hline \multirow{2}{*}{ Method } & \multicolumn{4}{c}{$J_{m 0}^{\mathrm{N}}$} \\
\cline { 2 - 5 } & 9 & 11 & 13 & 15 \\
\hline Gauss24 & 0.670064 & 0.717834 & 0.754797 & 0.785894 \\
Gauss48 & 0.670039 & 0.717804 & 0.754640 & 0.783692 \\
Gauss96 & 0.670038 & 0.717802 & 0.754638 & 0.783689 \\
Molin & 0.670038 & 0.717802 & 0.754638 & 0.783689 \\
$(2001)$ & & & & \\
\hline
\end{tabular}

Table 2 Convergence of $J_{m 0}^{\mathrm{D}}$ factors with Gauss integration order

\begin{tabular}{ccccc}
\hline \multirow{2}{*}{ Method } & \multicolumn{4}{c}{$J_{m 0}^{\mathrm{D}}$} \\
\cline { 2 - 5 } & 9 & 11 & 13 & 15 \\
\hline Gauss24 & 0.717680 & 0.742460 & 0.796973 & 0.831546 \\
Gauss48 & 0.717679 & 0.762457 & 0.796699 & 0.823468 \\
Gauss96 & 0.717672 & 0.762457 & 0.796699 & 0.823468 \\
Molin et & 0.717619 & 0.762457 & 0.796699 & 0.823468 \\
al. (2002) & & & & \\
\hline
\end{tabular}

Based on the numerical method, the estimation of the resonant frequencies is analyzed. Table 3 gives 
the frequencies for the first nine symmetric longitudinal modes in which there is no variation across the gap and they are obtained from Eq. (23) (designated as Theory ${ }^{\mathrm{N}}$ ), Eq. (24) (designated as Theory ${ }^{\mathrm{D}}$ ) and the full 3D calculation for beam seas based on present panel method codes. Table 4 shows resonant frequencies as predicted by the theory for the longitudinally symmetric $n=1$ modes. In Table 3 , the missing numerical results of the panel method for the modes $(11,0)$ and $(13,0)$ are because they are close to the frequencies for the modes $(7,1)$ and $(5,1)$. As a result, the former two modes are superimposed.

Overall, it may be seen that the simple semianalytical theory gives very close predictions of the resonant frequencies identified from 3D potential panel method, and the difference between $J_{m 0}^{\mathrm{N}}$ and $J_{m 0}^{\mathrm{D}}$ is reduced for the higher mode fluid resonance. That is to say, the influence of the boundary conditions at the end boundary becomes less prominent for higher resonant modes.
Fig. 8 shows the resonant modes of the wave elevations along the centerline of the gap in beam seas. These figures correspond to six modes, and the modes are symmetric longitudinally and uniform in the transverse direction. They confirm that the longitudinal variations of the elevations are indeed of type $(m, 0)$.

\section{Results of Wigley \& Barge}

The experiments investigating problems arising from the side-by-side arrangement of a modified Wigley model (which will be referred to as ship- $a_{1}$ ) and a rectangular barge model (which will be referred to as ship- $b_{1}$ ) were carried out by Kashiwagi et al. (2005). Both models are $L=2.0 \mathrm{~m}$ in length, $B=0.3 \mathrm{~m}$ in breadth, $d=0.125 \mathrm{~m}$ in draft and set in beam waves with two separation distances $D_{y}=$ $1.097 \mathrm{~m}$ and $1.797 \mathrm{~m}$.

Table 3 Resonant frequencies ( $\mathrm{rad} / \mathrm{s})$ for elevations in gap between two barges: $\boldsymbol{n}=\mathbf{0}$ mode

\begin{tabular}{|c|c|c|c|c|c|c|c|c|c|c|}
\hline \multirow{2}{*}{$B_{\mathrm{g}}(\mathrm{m})$} & \multirow{2}{*}{ Method } & \multicolumn{9}{|c|}{ Resonant frequency, $\omega_{\mathrm{e}}$} \\
\hline & & 1,0 & 3,0 & 5,0 & 7,0 & 9,0 & 11,0 & 13,0 & 15,0 & 17,0 \\
\hline \multirow{3}{*}{0.116} & Theory $^{N}$ & 5.9149 & 6.9639 & 8.2128 & 9.4628 & 10.6407 & 11.7318 & 12.7421 & 13.6830 & 14.5650 \\
\hline & Theory $^{\mathrm{D}}$ & 5.5564 & 6.8739 & 8.1799 & 9.4501 & 10.6357 & 11.7298 & 12.7413 & 13.6826 & 14.5649 \\
\hline & Present & 5.82 & 6.94 & 8.14 & 9.34 & 10.54 & 11.52 & 12.55 & 13.69 & 14.50 \\
\hline \multirow{3}{*}{0.200} & Theory $^{N}$ & 5.5114 & 6.7533 & 8.1184 & 9.4234 & 10.6249 & 11.7256 & 12.7397 & 13.6820 & 14.5647 \\
\hline & Theory $^{\mathrm{D}}$ & 5.0542 & 6.6359 & 8.0759 & 9.4072 & 10.6186 & 11.7231 & 12.7388 & 13.6816 & 14.5645 \\
\hline & Present & 5.34 & 6.62 & 7.90 & 9.18 & 10.42 & 11.47 & - & 13.61 & 14.45 \\
\hline \multirow{3}{*}{0.300} & Theory $^{\mathrm{N}}$ & 5.2114 & 6.6102 & 8.0598 & 9.4008 & 10.6164 & 11.7224 & 12.7385 & 13.6825 & 14.5645 \\
\hline & Theory $^{\mathrm{D}}$ & 4.6796 & 6.4704 & 8.0092 & 9.3815 & 10.6089 & 11.7195 & 12.7374 & 13.6811 & 14.5643 \\
\hline & Present & 4.94 & 6.38 & 7.66 & 9.10 & 10.14 & - & 12.24 & 13.63 & 14.36 \\
\hline
\end{tabular}

Table 4 Resonant frequencies ( $\mathrm{rad} / \mathrm{s})$ for elevations in gap between two barges: $n=1$ mode

\begin{tabular}{|c|c|c|c|c|c|}
\hline \multirow{2}{*}{$B_{\mathrm{g}}(\mathrm{m})$} & \multirow{2}{*}{ Method } & \multicolumn{4}{|c|}{ Resonant frequency, $\omega_{\mathrm{e}}$} \\
\hline & & 1,1 & 3,1 & 5,1 & 7,1 \\
\hline \multirow{3}{*}{0.116} & Theory $^{\mathrm{N}}$ & 16.3088 & 16.3801 & 16.5200 & 16.7234 \\
\hline & Theory $^{\mathrm{D}}$ & 16.3088 & 16.3801 & 16.5200 & 16.7234 \\
\hline & Present & 16.07 & 16.18 & 16.34 & 16.62 \\
\hline \multirow{3}{*}{0.200} & Theory $^{\mathrm{N}}$ & 12.4402 & 12.5985 & 12.8982 & 13.3121 \\
\hline & Theory $^{\mathrm{D}}$ & 12.4394 & 12.5979 & 12.8977 & 13.3118 \\
\hline & Present & 12.35 & 12.56 & 12.81 & 13.27 \\
\hline \multirow{3}{*}{0.300} & Theory $^{\mathrm{N}}$ & 10.2094 & 10.4879 & 10.9849 & 11.6248 \\
\hline & Theory ${ }^{\mathrm{D}}$ & 10.2028 & 10.4832 & 10.9819 & 11.6231 \\
\hline & Present & 10.36 & 10.54 & 11.39 & 11.81 \\
\hline
\end{tabular}


The mesh of these vessels is presented in Fig. 9, on which a rectangular zone (damping zone) between the vessels is shown as well. The experiment conducted first examined the forced heave oscillation tests, with ship- $a$ oscillated and ship- $b$ fixed. The second experiment was the measurement of the first-order wave-exciting forces in plane progressive waves with both ships completely fixed, corresponding to the diffraction problem. Here analysis of both cases is conducted, with the purpose of investigating the validation of present potential theory with an artificial damping force and the application of Molin's semi-analytical method to estimate the resonant frequencies.

\subsection{Analysis of added mass and damping coefficients}

The Wigley hull was forcibly oscillated in heave and the Barge fixed with separation distance $D_{y}=1.797 \mathrm{~m}$ is first surveyed. During Kasiwagi (2005)'s experiments, researchers measures manner in which the heave and sway added mass and damping coefficients as a result of the heave motion of Wigley-hull itself. The results of numerical computation with three values of parameter $\varepsilon=0,0.008$, and 0.016 are noted in Fig. 10 together with those of the measurements, against wave frequency $k_{0} L$. The results of Xiang (2013) predicted by using the Rankine panel method are also given. The added masses are nondimensional by the displaced mass of the Wigley ship $\rho \nabla_{a 1}$ and the damping by $\rho \nabla_{a 1} \omega$.

It can be seen from Fig. 10 that the numerical results from conventional methods are in agreement with the measurements, with the exception of those around the resonant peaks. The curves with dissipate parameter $\varepsilon=0.008$ are very similar close to the measurements. These also confirm the importance of the viscous damping effect on the strong hydrodynamic interaction and the validation of present potential method with an appropriate damping parameter. However, for added mass it seems that the no-lid case may yield closer predictions for some frequencies (such as $k_{0} L=12.68,16.66$ ); meanwhile, results obtained using the present method with dissipate parameter $\varepsilon=0.008$ agree well with experiment method for other resonant frequencies (such as

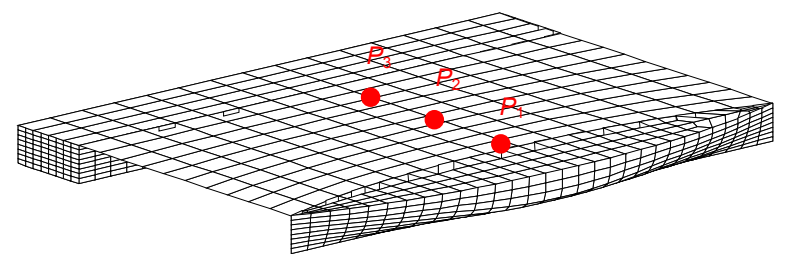

Fig. 9 Computational grid for Wigley, Barge, and gap inbetween of them
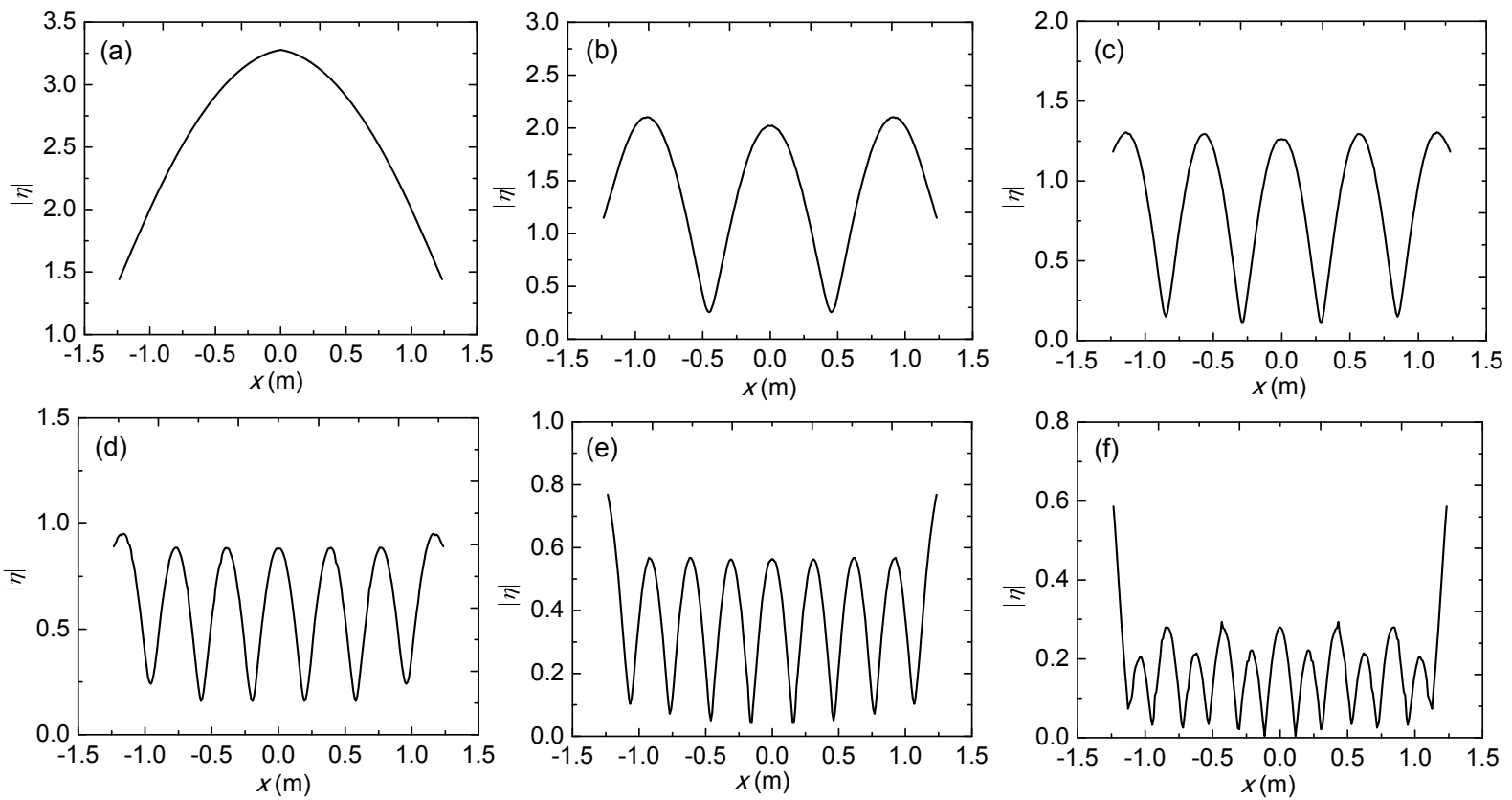

Fig. 8 Wave elevation along the gap in beam seas with $D_{y}=0.716 \mathrm{~m}$

(a) $m=1$; (b) $m=3$; (c) $m=5$; (d) $m=7$; (e) $m=9$; (f) $m=13$ 
$\left.k_{0} L=13.37,17.35\right)$. This may be due to the fact that added mass is the reaction force on the hull in phase with hull oscillations, and thus is less sensitive to viscous effects at some frequencies.

As can be seen in Fig. 10 and Fig. 11, the locations of the peaks of the damping coefficient are coincident with those of radiation elevation of $P_{1}$. However, resonant frequencies of the added mass are at slight variance with these results. This is due to different characteristic behaviors of the radiation coefficients in the vicinity of the resonant frequencies: the damping coefficient exhibits delta functionlike behavior and the added mass usually experiences first a maximum and then a minimum roughly centered on the damping maximum.
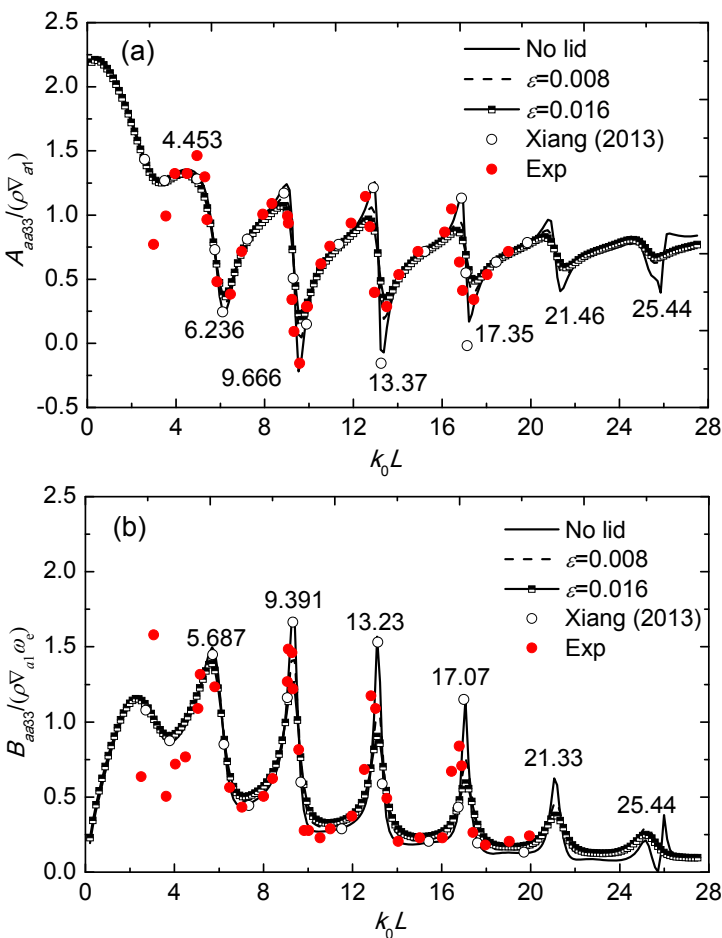

Fig. 10 Added mass (a) and damping (b) of Wigley with $D_{y}=1.797 \mathrm{~m}$

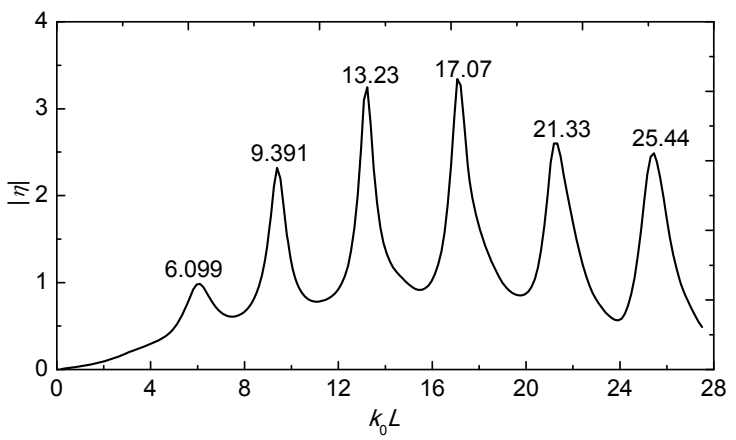

Fig. 11 Radiation elevation of $P_{1}$ with $D_{y}=1.797 \mathrm{~m}$

\subsection{Analysis of the first-order wave forces}

The excitation forces on the same WigleyBarge system with $D_{y}=1.097$ and beam seas are focused: the Wigley hull is on the weather side, and the Barge is on the shadow side. Amplitudes of the sway and heave excitation force on the Barge are shown in Fig. 12. The force amplitudes have been non-dimensionalized by $\rho g \zeta A_{\mathrm{wb}}$, where $A_{\mathrm{wb}}$ is the waterplane area of ship- $b_{1}$. It can be seen that numerical results with $\varepsilon=0.008$ may be generally in good agreement with the model test results and many repeated peaks of sway and heave forces can also be recorded. However, some discrepancies still occur in the low frequency range where the towing tank may not be wide enough to avoid the wall effects.
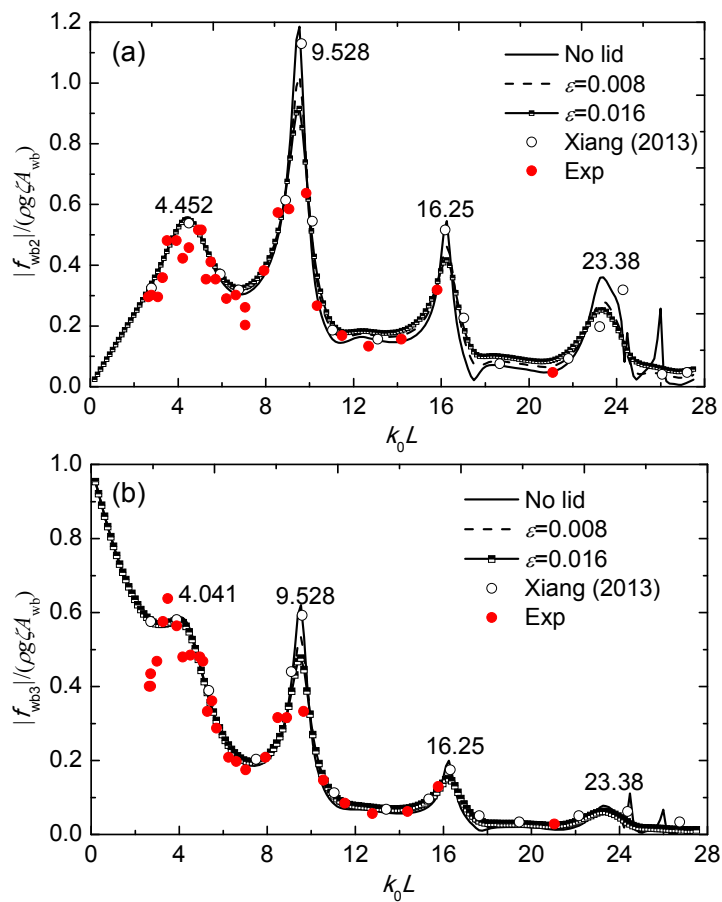

Fig. 12 Wave forces of barge ship at beam seas with $D_{y}=1.097 \mathrm{~m}$ : (a) sway forces; (b) heave forces

Fig. 13 shows the wave elevation of $P_{3}$ against $k_{0} L$, from which repeated peaks can be seen, and the corresponding frequencies are almost identical to those of wave forces. This indicates that a correlation exists between fluid and force resonance.

\subsection{Analysis of resonant frequencies}

As explored in section 4.4, the resonance frequencies can be estimated by Molin's simplified 
theory. Here, the Wigley hull is approximated as an identical barge ship, thus Eq. (21) and Eq. (22) may be used. The calculated resonant frequencies of "longitudinal sloshing mode" for the Wigley-Barge side-by-side system are presented in Table 5 . The simplified estimated results of $k_{0} L=(2 m-1) \pi$ proposed by Xiang (2013) and Zhu et al. (2008) are also listed. Overall, it may be observed that the simple, semi-analytical theory yields close predictions of the resonant frequencies identified from present 3D diffraction analysis. In addition, the equations corresponding to Neumann Conditions give better agreement with predicted resonant frequencies based on 3D panel method than other equations.

There follows further comparison of different methods to predict resonant frequencies. Table 6 gives the predicted resonant frequencies at different separations, such as $D_{y}=1.2 \mathrm{~m}, 1.5 \mathrm{~m}$, and $1.6 \mathrm{~m}$. It can be seen that the resonant frequencies tend to decrease with the increase of gap width, which mimic the results of the two barges system. However, the

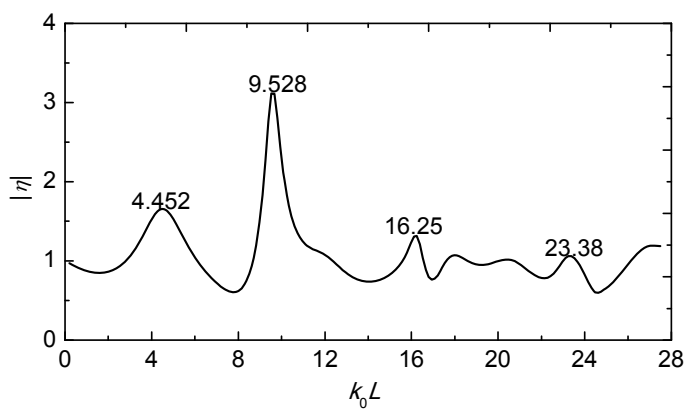

Fig. 13 Wave elevation of $P_{3}$ at beam seas with $D_{y}=1.097 \mathrm{~m}$ discrepancies of fluid resonant frequencies predicted by semi-analytical methods and present 3D diffraction analysis are more obvious with the increase of separation distances. This may be caused by the Wigley hull approximation.

Generally speaking, the Molin's simplified theory can provide an accurate estimation of resonant frequencies and be a practical tool for analyzing the fluid resonant phenomena of gaps in-between two floating bodies.

\section{Conclusions}

In this study, numerical simulations of fluid and force resonance in narrow gaps in-between two floating structures are conducted based on potential flow theory taking the effect of fluid viscosity by including the viscous damping force. Furthermore, the methods used to predict the resonant frequencies are introduced and their practical applications for ship

Table 5 Resonant frequencies of "longitudinal sloshing mode" for elevation in gap between two barges with $D_{y}=1.097 \mathrm{~m}\left(n=0\right.$ mode, $\left.k_{0} L\right)$

\begin{tabular}{ccccc}
\hline \multirow{2}{*}{ Method } & \multicolumn{4}{c}{ Resonant frequency } \\
\cline { 2 - 5 } & 1,0 & 3,0 & 5,0 & 7,0 \\
\hline Theory $^{\mathrm{N}}$ & 4.571 & 9.933 & 15.928 & 22.487 \\
Theory $^{\mathrm{D}}$ & 2.784 & 8.929 & 15.392 & 21.819 \\
$k_{0} L=(2 m-1) \pi$ & 3.142 & 9.425 & 15.708 & 21.991 \\
Present & 4.452 & 9.528 & 16.250 & 23.380 \\
\hline
\end{tabular}

Table 6 Resonant frequencies of "longitudinal sloshing mode" for elevation in gap between two barges $(n=0$ mode, $\left.k_{0} L\right)$

\begin{tabular}{ccccccc}
\hline \multirow{2}{*}{$D_{y}(\mathrm{~m})$} & \multirow{2}{*}{ Method } & \multicolumn{5}{c}{ Resonant frequency } \\
\cline { 3 - 6 } & & 1,0 & 3,0 & 5,0 & 7,0 & 9,0 \\
\hline \multirow{3}{*}{1.2} & Theory $^{\mathrm{N}}$ & 5.581 & 10.402 & 16.131 & 22.618 & 28.545 \\
& Theory $^{\mathrm{D}}$ & 3.928 & 9.637 & 15.734 & 21.978 & 28.259 \\
& $k_{0} L=\left(2 m^{-1}\right) \pi$ & 3.142 & 9.425 & 15.708 & 21.991 & 28.274 \\
& Present & 4.351 & 8.706 & 14.605 & 20.915 & 26.677 \\
\hline \multirow{3}{*}{1.5} & Theory & 5.054 & 10.142 & 16.017 & 22.551 & 28.517 \\
& Theory $^{\mathrm{N}}$ & 3.345 & 9.278 & 15.561 & 21.898 & 28.223 \\
& $k_{0} L=\left(2 m^{-1}\right) \pi$ & 3.142 & 9.425 & 15.708 & 21.991 & 28.274 \\
& Present & 3.630 & 7.059 & 11.449 & 20.778 & 25.579 \\
\hline \multirow{3}{*}{1.6} & Theory & 4.939 & 10.089 & 15.995 & 22.538 & 28.512 \\
& Theory & 3.215 & 9.197 & 15.523 & 21.880 & 28.215 \\
& $k_{0} L=\left(2 m^{-1}\right) \pi$ & 3.142 & 9.425 & 15.708 & 21.991 & 28.274 \\
& Present & 3.355 & 6.785 & 10.626 & 19.406 & 23.796 \\
\hline
\end{tabular}


hulls are investigated. Some of our key findings are outlined as follows:

1. The numerical results from present potential theory with appropriate damping coefficients produce results which corroborate experimental data both for forces and wave elevation. The accuracy of the predicted resonant wave height and force can thus be greatly improved. In addition, the dissipate parameter can be calibrated by comparing the wave elevation results of potential theory to those of measurements in experiments or the viscous fluid model, and the latter is more convenient and practical.

2. With regards to the two side-by-side Barges system and side-by-side Wigley-Barge system, the frequencies and magnitude of the peaks in the wave elevations and forces are generally dependent on the spacing and body's motion. With an increase of gap width, the peaks occur at lower frequencies.

3 . The semi-analytical model gives reliable predictions of the peak frequencies calculated by the present 3D panel method at different lateral clearances of the two Barges system. Additionally, the fluid resonant mode in the gap corresponding to each resonant frequency has also been confirmed. This model can be used to predict the peak frequencies for ship hulls with complex geometries, such as Wigley $\&$ Barge system.

Generally speaking, the Molin's simplified theory can give a reliable estimation of resonant frequencies and serve as a practical tool to analyze the fluid and force resonant phenomena of gaps inbetween two Barge systems and the Wigley \& Barge systems. Future studies will focus on the fluid and force resonance of two structures advancing in waves with forward speed. These cases are more complicated but are very important for advancing the underway replenishment operation.

\section{References}

Buchner, B., Dijk, A.V., Wilde, J.D., 2001. Numerical multiple-body simulation of side-by-side mooring to an FPSO. Proceedings of the 11th ISOPE, International Society of Offshore and Polar Engineering, Copertino, USA, p.343-353.

Chen, X.B., 2011. Offshore hydrodynamics and applications. The IES Journal Part A: Civil \& Structural Engineering, 4(3):124-142.

Guevel, P., 1982. Le probleme de diffraction-radiationPremiere partie: theoremes fondmentaux. Ecole Supéri- eure de Mécanique de Nantes (ENSM), University of Nantes, Nantes, France (in French).

Iwata, H., Saitoh, T., Miao, G.P., 2007. Fluid resonance in narrow gaps of very large floating structure composed of rectangular modules. Proceedings of the 4th International Conference on Asia and Pacific Coasts, Beijing, China, p.815-826.

Kashiwagi, M., 2007. 3-D calculation for multiple floating bodies in proximity using wave interaction theory. Proceedings of the 17th International Conference on Offshore and Polar Engineering, Lisbon, Portugal.

Kashiwagi, M., Endo, K., Yamaguchi, H., 2005. Wave drift forces and moments on two ship arranged side by side in waves. Ocean Engineering, 32:529-555.

Koo, B.J., Kim, M.H., 2005. Hydrodynamic interactions and relative motions of two floating platforms with mooring lines in side-by-side offloading operation. Applied Ocean Research, 30:1-16.

Lewandowski, E.M., 2008. Multi-vessel seakeeping computations with linear potential theory. Ocean Engineering, 35(11-12):1121-1131. [doi:10.1016/j.oceaneng.2008.04. 011]

Lu, L., Teng, B., Cheng, L., et al., 2011a. Modelling of multibodies in close proximity under water waves-fluid resonance in narrow gaps. Science China Physics, Mechanics and Astronomy, 54(1):16-25. [doi:10.1007/s11433-0104194-8]

Lu, L., Teng, B., Sun, L., et al., 2011b. Modelling of multibodies in close proximity under water waves-fluid forces on floating bodies. Ocean Engineering, 38(13): 1403-1416. [doi:10.1016/j.oceaneng.2011.06.008]

Miao, G.P., Ishida, H., Saitoh, T., 2000. Influence of gaps between multiple floating bodies on wave forces. China Ocean Engineering, 14(4):407-422.

Molin, B., 2001. On the piston and sloshing modes in moonpools. Journal of Fluid Mechanics, 430:27-50. [doi:10.1017/S0022112000002871]

Molin, B., Remy, F., Kimmoun, O., et al., 2002. Experimental study of the wave propagation and decay in a channel through a rigid ice-sheet. Applied Ocean Research, 24(5):247-260. [doi:10.1016/S0141-1187(03) 00005-1]

Molin, B., Remy, F., Camhi A., et al., 2009. Experimental and numerical study of the gap resonances in-between two rectangular barges. 13th Congress of International Maritime Association of Mediterranean, Istanbul, Turkey.

Newman, J.N., 2003. Application of Generalized Modes for the Simulation of Free Surface Patches in Multiple Body Interactions. WAMIT Consortium Report.

Newman, J.N., Sclavounos, P.D., 1988. The computation of wave loads on large offshore structures. BOSS Conference, Trondheim, Norway.

Pauw, W.H., Huijsmans, R.H.M., Voogt, A., 2007. Advances in the hydrodynamics of side-by-side moored vessels. Proceedings of the 26th International Conference on Offshore Mechanics and Arctic Engineering, San Diego, USA, 2007. 
Recktenwald, G.W., 2000. Numerical Methods with MATLAB: Implementations and Applications. PrenticeHall, NJ.

Rippol, T., 2004. Navires 'a couples. Rapport d'essais. C04.2.013, GIS HYDRO, Océanide (in French).

Saitoh, T., Miao, G.P., Ishida, H., 2006. Theoretical analysis on appearance condition of fluid resonance in a narrow gap between two modules of very large floating structure. Proceedings of the 3rd Asia-Pacific Workshop on Marine Hydrodynamics, Beijing, China, p.170-175.

Sun, L., Taylor, P.H., Taylor, R.E., 2008. First and second order wave effects in narrow gaps between moored vessels. Marine Operations Specialty Symposium, Singapore.

Sun, L., Taylor, R.E., Taylor, P.H., 2010. First and second order analysis of resonant waves between adjacent barges. Journal of Fluids and Structures, 26(6):954-978. [doi:10.1016/j.jfluidstructs.2010.06.001]

Telste, J.G., Noblesse, F., 1986. Numerical evaluation of the Green function of water-wave radiation and diffraction. Journal of Ship Research, 30(2):69-84.

Wang, C.Z., Wu, G.X., 2008. Analysis of second-order resonance in wave interactions with floating bodies through a finite method. Ocean Engineering, 35(8-9):717-726. [doi:10.1016/j.oceaneng.2008.02.004]

Xiang, X., 2013. Maneuvering of Two Interacting Ships in Waves. PhD Thesis, Norwegian University of Science and Technology, Norwegian.

Yao, C.B., Dong, W.C., 2014. A fast integration method for translating-pulsating source Green's function in Bessho form. Journal of Zhejiang University-SCIENCE A (Applied Physics \& Engineering), 15(2):108-119. [doi:10. 1631/jzus.A1300209]

Zhu, R.C., Miao, G.P., You, Y.X., 2005. Influence of gaps between 3-D multiple structures on wave forces. Journal of Hydrodynamics Series B, 172:141-147.

Zhu, R.C., Zhu, H.R., Miao, G.P., 2008. Influences on hydrodynamics of multiple floating structures with small gap in between. Journal of Shanghai Jiaotong University, 42(8):1238-1242 (in Chinese).

\section{中文概要}

题 目: 近距两浮体间流体共振分析

目 的: 基于三维线性势流理论, 通过在近距两浮体间 的自由液面上引入 “粘性耗散系数”, 建立计及 粘性影响的波浪中近距两浮体水动力干扰效应 分析数值计算方法, 以准确分析两浮体的干扰 力及浮体间液面升高; 并探讨 “粘性耗散系 数”的确定方法、两浮体间流体共振频率及共 振模式的数值计算方法。

方 法: 1. 通过理论分析, 在三维线性势流理论基础 上，引入流体 “粘性耗散” (公式 2、10 12、18 和 19) 以准确模拟近距两浮体波浪中的水动力 及浮体间液面抬升; 2. 采用雷诺应力平均方程 （RANSE）方法或试验方法确定 “粘性耗散系 数” (图 2 和 3 ); 3. 采用数值计算和理论分析的 方法给出近距两浮体间的流体共振模式（图 8) 及共振频率估算方法 (公式 21 25)。

结 论: 1. 采用三维线性势流理论并引入流体 “粘性耗 散系数” 可较为准确地计算得到近距两浮体波 浪作用下的受力及浮体间液面抬升; “粘性耗散 系数”可通过 RANSE 方法或试验方法获得; 2 . 相比于其它因素 (浪向角等), 近距两浮体间的 干扰效应受横向间距影响较大； 3. 采用类似于 “月池” 中流体共振频率分析方法获得的共振 频率计算公式可用于估算近距两浮体间的流体 共振频率，同时不同共振频率时浮体间的流体 共振模式得到了数值计算结果的验证。

关键词: 流体共振; 水波; 边界元方法; 人工阻尼力; 晃 荡模式 